\title{
EDITORIAL
}

\section{Evidence-Based Neurotherapeutics: A Spectrum of Evidence}

\section{INTRODUCTION}

The evidence-based medicine (EBM) revolution drastically altered the sources of information that doctors turn to when deciding how to treat their patients. The central tenet of EBM is that the scientifically conducted study-evidence-represents the most reliable information for medical decision-making. This new way of thinking places new demands on clinicians who must understand the uses and limitations of the various types of evidence at their disposal. It also places demands on the evidence itself to be of high quality and to address the issues important to the researchers, clinicians, and policy makers.

Traditionally, the purpose of EBM teachings is for bedside decision-making and for assisting providers in caring for their patients. In this issue of NeuroRx ${ }^{\circledR}$, we take a much broader perspective of evidence. We explore not only the evidence that guides clinical decision-making, but also the evidence generated along the pathway that begins at the scientists' "benchside" and is implemented at the policy researchers' "curbside" (FIG. 1). By examining this process in depth, we hope to give our readers a new perspective that will allow them to better use the ever-increasing mass of information at their disposal. Here are examples of questions addressed in this issue:

1) What is the evidence needed to take promising agents from animals and begin testing them in humans?

2) What is the evidence being generated from biomar-

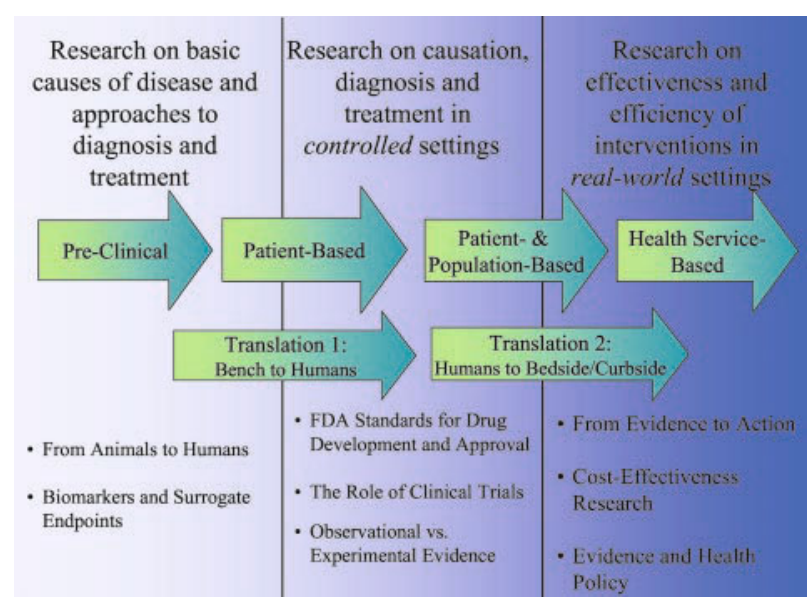

FIG. 1. Spectrum of neurotherapeutic evidence. kers and how can it be used to help understand and treat neurological disease?

3) What are the evidentiary standards used by the Food and Drug Administration (FDA) to approve drugs for neurological disease?

4) What types of evidence does the National Institute of Neurological Disorders and Stroke (NINDS) see as funding priorities?

5) What is the type of evidence being generated from clinical trials and how can it be used no only for drug approval but for clinical-decision making?

6) How does evidence from nonrandomized studies, such as observational studies, differ from the evidence generated from randomized studies, and under what circumstances should we rely on observational evidence to guide clinical decision-making?

7) What is the "evidence-practice" gap and what are the evidence-based methods to close it?

8) What evidence is being generated from cost-effectiveness research and how can this evidence be used to guide clinical decision-making, drug formulary decisions, and insurance-policy decisions?

9) How can research evidence be used to influence policy decisions at the local, state, or national level?

10) What is "evidence-based ethics" and why is it increasingly important in neurotherapeutics research?

The audiences to interpret and act upon the evidence being generated include: basic scientists, regulators, clinical researchers, practicing clinicians, health service researchers, physician, health systems, insurance companies, politicians, and patients. The basic EBM approach, however, remains the same for stakeholders: 1) find the best source of available evidence, 2) critically appraise the evidence, and 3) apply the new information to basic science/translational/patient-oriented research initiatives, to patient care, or to policy decisions. ${ }^{a}$

\section{A SPECTRUM OF EVIDENCE}

The first step in this process is basic biomedical research. This type of research often asks the question: "What are the underlying mechanisms causing disease and what are rational approaches to diagnosis and treatment based on these mechanisms?" This research converts basic insights into applications that can, in principal, be applied to patients. This is also the step at which 
the basic tools for performing clinical experiments are developed.

The next point along the pathway takes the insights gained from applications in preclinical models and converts them into clinical experiments. This type of research addresses the question: "How can disease be diagnosed, prevented or treated under controlled settings?" Much of the "patient-oriented" research enterprise is geared toward generating this kind of evidence. The standard research paradigm for this stage is the randomized, controlled trial. Alternatively, observational studies in large populations of patients address the applicability of new treatments that are beyond the scope of randomized clinical trials.

The last phase in the process is health services research (HSR). HSR is concerned with taking the results of clinical studies and implementing them in clinical practice. This type of research is equally likely to evaluate physicians and organizations as it is to evaluate patients, and asks the question: "What works in a realworld setting and what does it cost?" HSR examines how people access health care, how much care costs, and what happens to patients as a result of this care. The main goals of HSR are to identify the most effective ways to organize, manage, finance, and deliver high-quality care, reduce medical errors, and improve patient safety. ${ }^{1}$

Two transition points along this spectrum of biomedical research link basic insights, clinical investigations, and improved healthcare. The first transition, between basic research and patient-oriented research, is often referred to as "translational research." 2 The second transition involves taking evidence generated from patientoriented research and producing tangible improvements in clinical practice and patient health. Although this would seem to be a relatively simple enterprise compared to the process of creating biomedical innovation, there are substantial obstacles integrating biomedical discoveries into routine clinical practice. ${ }^{3}$ This lag between innovation and implementation has been referred to as the "practice gap."

We have organized the issue into the following four sections: 1) Evidence from Basic Biomedical Research/ Translational Research, 2) Evidence from Human Studies, 3) Evidence to Close the Practice Gap, and 4) Enabling Factors. Improving the flow of information along this research evidence highway will require the collaboration of many individuals and will demand a multifaceted approach between researchers, providers, funders, and regulators. Open communication among all of these groups is essential for evidence to reach its intended target and for the evidence that is generated to be relevant to the problems faced by the various stakeholders today. This will require an increasing collaboration by basic scientists, clinical scientists, practitioners, policy analysts, and patients at all stages of the process of generation and dissemination of evidence, which will increase the likelihood of better health outcomes for patients with neurological disease.

\section{Section I: Evidence from basic biomedical research/ translational research}

In the first section of this issue, Steven M. Hersch and Robert J. Ferrante, in Translating Therapies for Huntington's Disease from Genetic Animal Models to Clinical Tri$a l s$, discuss the type of evidence required to bring therapies from the lab into humans and describe criteria for judging the quality of preclinical evidence from a Huntington's disease mouse paradigm. The technologies required for this type of research include microarrays, genetically engineered cells and organisms, proteomics, and high-throughput drug testing systems. Andrew Feigin, in Evidence from Biomarkers and Surrogate Endpoints, discusses the opportunities that reside in the biomarker revolution and offers an epistemology of surrogate outcome measures. These contributions cover the basic science underpinnings required to bring basic insights to human studies. These studies supply evidence about pathomechanisms, basic biology, diagnosis, prognosis, drug development, and potentially surrogate endpoints to be used as substitutes for clinical endpoints in clinical trials.

\section{Section II: Evidence from human studies}

In the next section, Russell Katz, the Director of the Division of Neuropharmacological Drug Products, in FDA: Evidentiary Standards for Drug Development and Approval, demystifies the regulatory standards and the type of evidence used for drug development and drug approval. Andrew Siderowf, in Evidence from Clinical Trials: Can We Do Better?, discusses the kinds of evidence being produced currently in clinical trials, and the potential to increase the relevance of clinical trials. John Concato, in Experimental Versus Observational Studies: What's the Evidence for a Hierarchy?, makes the case for evidence from observation studies and how it differs from evidence from randomized trials. Although the gold standard evidence is the randomized controlled trial, there are circumstances in which randomization is not feasible in neurology. In fact, the vast majority of what we do in neurological care will not be based in randomized trials, and Dr. Concato discusses the roles, uses, and limitation of observational evidence in making treatment decisions.

\section{Section III: Evidence to close the practice gap}

Gary G. Gronseth, in From Evidence to Action, discusses why the passive diffusion of evidence through scientific journals and continuing medical education offerings is often not enough to change practice. He reviews proactive methods to promote patient, provider, and organizational behavior change to be in line with the evidence available. He discusses evidence-processing 
techniques, traditional EBM techniques, and methods to combine sources of evidence to improve clinical decision-making, including systematic reviews, Cochrane reviews, and clinical practice guidelines. Katia Noyes and Robert Holloway, in Evidence from Cost-Effectiveness Research, discuss the purpose, methods, and interpretation of cost-effectiveness research in neurological diseases. They review how evidence from cost-effectiveness research differs from more familiar evidence used in patient-oriented research, but how it can be used for decision-making purposes. In doing so, they discuss how modeling techniques are commonly used in other policy arenas, including environmental policy and defense policy, and how models can be valuable for health-care decision makers. Gary M. Franklin et al., in PolicyRelevant Research: When Does It Matter?, discuss evidence in the research-to-policy connection. In the context of their work with the system for providing healthcare for work-related injuries in Washington state, he describes the critical role that evaluative research plays in identifying policy problems, developing a solution, and informing the debate and decisions to take policy action. The decision-makers, however, are often different than clinicians and patients, and include hospital committees, managed care, government, and employers. ${ }^{1}$

\section{Section IV: Enabling factors}

To allow basic science insights to enter clinical testing, and to close the "practice gap," there will need to be increased funding, an increase in support for training and mentoring researchers, an increase in cooperation among diverse stakeholders, and efforts to minimize potential conflicts of interest. Bernard Ravina, from the National Institutes of Neurological Disorders and Stroke, and his colleagues, in Funding Evidence: the National Institute of Neurological Disorders and Stroke Clinical Trials Program, provide an overview of the NINDS funding pattern and priorities. They review descriptive informa-

\footnotetext{
${ }^{a}$ Key Words: Evidence-based medicine, therapy, health services research, patient-oriented research, health policy.
}

tion about the current research being funded, by treatment groupings, and by phase of trial, and how the NINDS sees itself functioning in the future: what its research priorities will be, how it might support infrastructure for collaborative group studies or provide other tangible assistance for investigators, and how it will increase funding for health services research. Finally, Scott Y. H. Kim, in Evidence-Based Ethics for Neurology and Psychiatry Research, discusses examples of important ethical issues in neurotherapeutics and the need for evidence-based ethics in both research and practice. Dr. Kim will address such "hot-button" issues as high-risk therapies, complicated conflicts of interest, and informed consent, especially in individuals with neurological disease and reduced decision-making capacity.

Our goal in this special focus issue of NeuroRx ${ }^{\circledR}$ is to lay out the key components in the process of creating and disseminating evidence for medical decision-making and creation of health policy. It is our hope that this stateof-the-art survey of evidence-based neurotherapeutics will provide a foundation for advances in the generation and application of evidence across the spectrum of research and clinical practice. Ultimately, these advances may lead to better approaches for managing patients with neurologic disease that will improve the health and wellbeing of our society.

Robert G. Holloway, M.D., MPH e-mail: robert.holloway@ctcc.rochester.edu

Andrew D. Siderowf, M.D., MSCE e-mail: asiderowf@pahosp.com

Guest Editors

\section{REFERENCES}

1. Siu A. The health services researcher, multiple identities. Health Serv Res 37:3-6, 2002.

2. Rosenberg RN. Translating biomedical research to the bedside: a national crisis and a call to action. JAMA 289:1305-1306, 2003.

3. Lenfant C. Shattuck lecture: clinical research to clinical practicelost in translation? N Engl J Med 349:868-874, 2003.

4. Berwick DM. Disseminating innovations in health care. JAMA 289: 1969-1975, 2003. 